\title{
COกE).(OES
}

CIÊNCIA E TECNOLOGIA

\section{IMPACTOS DO PROGRAMA BRASIL CARINHOSO NA EDUCAÇÃO, SAÚDE E RENDA}

\author{
Nayara Magda Gomes Barbosa da Costa ${ }^{1}$, Francisco Herbert Lima Vasconcelos ${ }^{1}$, \\ Tiago da Costa Silva Barbosa ${ }^{2}$, NATÃ da Costa Silva ${ }^{1}$ \\ ${ }^{1}$ Universidade Federal do Ceará - UFC \\ ${ }^{2}$ Instituto Federal de Educação, Ciência e Tecnologia do Piauí - IFPI \\ <nayaramenfer@hotmail.com>.<herbert@virtual.ufc.br>. <tiago.silva@ifpi.edu.br $>$ \\ $<$ natancsilva@hotmail.com> \\ DOI: $10.21439 /$ conexoes.v15i0.2098
}

\begin{abstract}
Resumo. O Brasil Carinhoso é um programa intersetorial de transferência de renda que visa ao fortalecimento integral da educação infantil no combate à desigualdade social. Devido ao seu caráter integral, inclui-se no programa ações nas áreas de educação, saúde e renda. Nesse contexto, o presente artigo trata-se de uma pesquisa bibliográfica que objetivou relatar os impactos do Programa Brasil Carinhoso na educação, saúde e renda da população beneficiada, baseado na hipótese de que o referido programa corrobora para melhoria das condições básicas de vida da população abrangida. Para isso, realizou-se uma análise bibliográfica nas publicações técnicas e científicas presentes nos portais SciELO e Google Scholar, bem como, em sites oficiais do governo federal brasileiro. Na análise foram consideradas as publicações cuja temática é objeto da presente pesquisa. No geral, observou-se que as ações do Brasil Carinhoso têm impactado positivamente as famílias beneficiárias, garantindo a redução da situação de extrema pobreza e melhorando o desenvolvimento infantil nos aspectos educacionais e de Saúde.
\end{abstract}

Palavras-chaves: Fortalecimento da educação infantil. Desenvolvimento social. Crescimento saudável. Políticas públicas. Efeitos positivos.

\section{BRASIL CARINHOSO PROGRAM IMPACTS ON EDUCATION, HEALTH AND INCOME}

\begin{abstract}
The Brasil Carinhoso is an intersectoral income transfer program, which aims at the integral strengthening of child education fighting social inequality. Due to its integral character, the program includes actions in education, health and income areas. In this context, the present article is a bibliographic research that aimed at reporting the impacts of this program within its action areas observing the benefited population based on the hypothesis that this program corroborates to improve the basic living conditions of the population included. For that, a bibliographic analysis was carried out in the technical and scientific publications on the SciELO and Google Scholar portal as well as on official websites of Brazilian federal government. The analysis considered publications whose theme is the subject of this research. Overall, it was observed that the actions of Brasil Carinhoso have positively impacted beneficiary families ensuring the reduction of the situation of extreme poverty and improving the child development in educational and health aspects.
\end{abstract}

Keywords: Strengthening of child education. Social development. Healthy growth. Public policy. Positive effects.

\section{INTRODUÇÃO}

De acordo com o Fundo Nacional de Desenvolvimento da Educação - FNDE (2017), o Programa Brasil Carinhoso (PBC) consiste na transferência automática de recursos financeiros da União aos Municípios e Distrito Federal com a finalidade de custear gastos com manutenção e desenvolvimento integral da educação infantil, tendo como base legal a Lei $\mathrm{N}^{\circ} 12.722$, de 3 de outubro de 2012. O programa foi criado para atender à parcela mais vulnerável da sociedade economicamente desprovida e envolve ações do Ministério da Educação, da Saúde e do Desenvolvimento Social e Combate à Fome (FONTANA; CAMPOS, 2017). 
Para Costa, Mafra e Bachtold (2014) o PBC destacase como a mais importante inovação do Plano Brasil Sem Miséria (BSM), uma vez que foi concebido numa perspectiva de atenção integral envolvendo aspectos do desenvolvimento infantil ligados à renda, educação e saúde. Entre as vantagens do PBC salienta-se o fortalecimento da educação infantil e o combate à desigualdade social. Para Fernandes (2014), proporcionar uma educação infantil de qualidade é o remédio mais eficaz para combater as diferenças sociais a longo prazo, sendo esse, o primeiro passo para quebrar o círculo de reprodução da desigualdade social. Haddad et al. (2014) afirmam que o PBC reforça a prioridade às crianças nas políticas para erradicação da pobreza. Ainda conforme os autores, o programa baseia-se nas evidências científicas que apontam a pobreza como um obstáculo ao desenvolvimento infantil e o baixo desenvolvimento infantil como fator de reprodução da pobreza.

Por se tratar de um programa intersetorial, o PBC tem influenciado na educação, saúde e renda das famílias beneficiadas. No aspecto da renda, o programa possibilitou a criação do benefício de superação da extrema pobreza adicional ao Programa Bolsa Família (PBF). Por meio desse novo benefício as famílias passaram a receber um valor complementar ao $\mathrm{PBF}$, necessário para que todos os membros pudessem superar a linha da extrema pobreza, sendo a quantia variável de acordo com a situação econômica de cada família.

Na educação, conforme Costa, Mafra e Bachtold (2014), o PBC dedicou-se à ampliação do acesso à creche, por meio do estímulo financeiro às prefeituras e ao Distrito Federal para a abertura de vagas, especialmente para a população mais pobre. O recurso financeiro transferido via PBC destina-se aos alunos com idade de zero a 48 meses que estejam devidamente matriculados em creches públicas ou conveniadas e cujas famílias sejam beneficiadas com o PBF (FNDE, 2017). $\mathrm{O}$ PBC estabelece os critérios para o repasse financeiro e a Resolução $N^{\circ} 1$, de 28 de novembro de 2014, da Secretaria de Educação Básica do Ministério da Educação, define as despesas permitidas.

Na saúde, as ações do PBC visam ao desenvolvimento saudável das crianças, principalmente na primeira infância. Para Viera et al. (2015) essa faixa etária merece atenção dos serviços de saúde, pois nela ocorrem modificações, que influenciam o desenvolvimento e a fase adulta. Segundo Costa, Mafra e Bachtold (2014), nos aspectos de saúde, o programa previne e trata alguns dos problemas que mais prejudicam o desenvolvimento na primeira infância. Ainda conforme os autores, o Ministério da Saúde, intermediado pelo PBC, expandiu a distribuição de doses de vitamina A e sulfato ferroso, bem como, passou a distribuir gratuitamente medicamentos para asma para o público alvo.

Nesse contexto, o presente estudo tem por objetivo relatar os impactos do Programa Brasil Carinhoso na educação, saúde e renda da população beneficiada. Partindo da hipótese de que o referido programa corroborou para melhoria das condições básicas de vida da população abrangida.

\section{FUNDAMENTAÇÃO TEÓRICA}

\subsection{Políticas Públicas de Transferência de Renda}

As políticas públicas podem ser compreendidas como ações governamentais que atendem grande parte da sociedade e busquem assegurar o bem-estar social, as necessidades fundamentais do indivíduo e os direitos de cidadania (MACEDO; NOIA; SANTOS C. E. R.A ND PINHEIRO, 2017), elas podem ser estabelecidas a nível federal, estadual e/ou municipal. No âmbito das políticas públicas, as demandas sociais são apresentadas aos dirigentes públicos por meio de grupos organizados (LOPES; AMARAL; CALDAS, 2008) e a partir disso são elaboradas estratégias de mitigação dos problemas diagnosticados.

Entre as políticas públicas, destacam-se nacionalmente aquelas que são destinadas à transferência de renda, pois por atenderem às necessidades básicas, elas possibilitam a redução da situação de extrema pobreza, principalmente nos países em desenvolvimento. Essas políticas são fundamentais para que a população de maior vulnerabilidade socioeconômica obtenha as condições mínimas necessárias de sobrevivência.

O desenvolvimento e implantação de estratégias de transferência de renda entre Estado e população não é uma ação atual e exclusiva do governo brasileiro. Para Diniz (2007) o desenvolvimento de programas de garantia de renda mínima não é recente e teve na Europa Ocidental as suas primeiras implantações. A partir da constituição dos Estados nacionais modernos, as ações de combate à fome e à pobreza passam a integrar a agenda das autoridades e ao longo do tempo passam a enfrentar o diagnóstico da grande pobreza e exclusão social com o desenvolvimento de políticas públicas específicas (PASE; MELO, 2017).

No Brasil, no final da década de 1990 e início da década de 2000, deram início articulações de programas de transferência de renda em nível federal, com o intuito de suprir as necessidades básicas de populações vulnerabilizadas (PETRINI; DIAS, 2013). Nesse contexto, em 2001, criou-se o Programa Bolsa Escola que configurava-se como um programa de renda mínima associado a ações socioeducativas, sendo coorde- 
nado pelo Ministério da Educação (CÓRDOVA; ALVES 2019). Além do Bolsa Escola foram criados também o Programa Bolsa Alimentação que visava fortalecer a nutrição da população pueril, sendo coordenado pelo Ministério da Saúde e o Programa Auxílio Gás, coordenado pelo Ministério de Minas e Energia, como ação compensatória pelo fim do subsídio ao gás de cozinha, conforme Córdova e Alves (2019). No início de 2004 foi estabelecido o Programa Bolsa Família $(\mathrm{PBF})$ destinado às famílias em situação de pobreza e extrema pobreza e que unificou os programas Bolsa Escola, Bolsa Alimentação e Auxílio Gás (DINIZ, 2007). Ao longo do tempo, benefícios adicionais ao PBF foram surgindo de acordo com as necessidades mais urgentes da população.

Atualmente, uma grande parcela da população brasileira é assistida pela política de transferência de renda, uma vez que diversos fatores têm corroborado para perda e/ou redução da renda familiar e consequentemente redução da qualidade de vida da população. Nesse contexto socioeconômico a população passou a depender mais das políticas assistencialistas do Estado, evidenciando a importância dessas ações na garantia das condições básicas de sobrevivência. Como exemplo, tem-se a grande quantidade de brasileiros que passou a depender financeiramente do programa de transferência de renda emergencial em decorrência da crise econômica desencadeada pela pandemia da Covid-19.

\subsection{Plano Brasil Sem Miséria (BSM)}

O plano BSM pode ser compreendido como um conjunto de ações governamentais no combate à extrema pobreza no Brasil, direcionado a população de maior vulnerabilidade socioeconômica. De acordo com a (SECRETARIA ESPECIAL DO DESENVOLVIMENTO SOCIAL, 2015), esse plano foi criado com o intuito de superar a extrema pobreza no país de forma integral. Por isso, diversos aspectos socioeconômicos foram considerados prioridades na elaboração do referido plano (SEDS, 2015). O BSM coordena mais de 70 programas, serviços e benefícios públicos e envolve vários ministérios, tendo iniciado suas atividades em 2011 (TRONCO; RAMOS, 2017).

Para o desenvolvimento do BSM e melhor direcionamento das ações e recursos financeiros, foram estabelecidas as linhas de pobreza e extrema pobreza para o país, tendo como base as linhas utilizadas pelo PBF. Embora existam críticas quanto aos critérios utilizados para a determinação das linhas de pobreza, ela representa uma estratégia positiva para a definição unificada da população brasileira pobre e extremamente pobre. Tronco e Ramos (2017) afirmam que a determinação da linha oficial de pobreza no BSM foi uma ação inovadora, uma vez que, os programas anteriores utilizavam critérios próprios não unificados para definir a pobreza no Brasil. Ainda de acordo com os autores, o MDS estabeleceu na época o valor de $\mathrm{R} \$ 70,00$ per capita para definir famílias em situação de extrema pobreza e o valor de $\mathrm{R}$ \$ 140,00 para definir as famílias em situação de pobreza.

Para ter acesso às famílias em situação de pobreza e extrema pobreza o Estado passou a buscá-las e incluílas no Cadastro Único de Programas Sociais (CADÚnico) que consiste em um grande cadastro da população brasileira de baixa renda, a partir do qual elas são inscritas em programas sociais. Essa ação foi denominada de Busca Ativa conforme afirmam Tronco e Ramos (2017). A Busca Ativa é uma ação presente em todo o plano e que objetiva levar o Estado onde o cidadão está, sem esperar que as pessoas mais pobres procurem o poder público (BACHTOLD, 2016). Embora tenha sido um dos principais desafios do BSM a ação Busca Ativa apresentou importantes resultados para a aplicação do plano.

Conforme Costa e Falcão (2014), as ações que compõem o BSM estão distribuídas em três grandes eixos de atuação. O primeiro eixo está relacionado às transferências de recursos financeiros às famílias de baixa renda para redução urgente da situação de extrema pobreza no país. Fazem parte desse eixo o PBF e o Benefício de Prestação Continuada (BPC), sendo o PBF o principal componente desse eixo (COSTA; FALCãO, 2014). Para Tronco e Ramos (2017) benefícios adicionais são incorporados ao PBF, caso verifique-se no núcleo familiar gestantes, crianças na primeira infância e crianças e adolescentes em idade escolar de até 15 anos. Entre os benefícios adicionais ao PBF, tem-se o PBC.

O segundo eixo do BSM visa melhorar a incorporação da população de baixa renda no mercado de trabalho urbano e rural. Para Costa et al.(2014) a inserção da população pobre no mundo do trabalho é precária, apresentando instabilidade e renda baixa. Ainda em consonância com os autores, isso pode ser consequência do baixo acesso à educação e a bons cursos de qualificação profissional. Nesse contexto, as estratégias adotadas nesse eixo na área urbana visam à qualificação profissional da população de baixa renda, para que os mesmos tenham melhores possibilidades de ingressarem e se manterem no mercado de trabalho, elevando-se assim a renda familiar. $\mathrm{Na}$ zona rural, as estratégias adotadas visam o fortalecimento da agricultura familiar garantindo principalmente assistência técnica e ações de fomento aos agricultores familiares de baixa renda.

Por fim, o terceiro e último eixo do BSM tem como 
objetivo aumentar o acesso e melhorar a qualidade dos serviços públicos de saúde, educação e assistência social, prestados à população de baixa renda principalmente das localidades mais pobres do país.

\section{METODOLOGIA}

$\mathrm{O}$ artigo trata-se de uma pesquisa bibliográfica de natureza básica com intuito de relatar os efeitos do PBC nos aspectos de educação, saúde e renda da população beneficiada. Pesquisas dessa categoria envolvem um conjunto ordenado de procedimentos de busca por soluções, atento ao objeto de estudo (LIMA; MIOTO 2007). Sendo assim, nesse artigo, realizou-se um estudo bibliográfico nas publicações técnicas e científicas presentes nos portais SciELO e Google Scholar, bem como, em sites oficiais do governo federal brasileiro. Nos portais pesquisados utilizou-se como critério de inclusão o tema dos trabalhos técnicos e científicos, sendo incluídos aqueles que abordassem a temática do presente estudo. Na busca pela bibliografia foram utilizados os termos: Brasil Carinhoso; fortalecimento da educação infantil; políticas públicas de transferência de renda; combate à desigualdade social brasileira; crescimento saudável e segurança alimentar; saúde, educação e renda no Brasil Carinhoso, deficiência de vitamina A em crianças, deficiência de ferro em crianças e saúde infantil. A pesquisa foi dividida em quatro etapas nas quais a primeira consistiu na busca da bibliografia nos portais e sites oficiais aplicando os termos de busca. A segunda contemplou a leitura dos títulos e resumos dos trabalhos encontrados, selecionando aqueles cuja temática é objeto dessa pesquisa. Na terceira etapa foi realizada leitura e análise detalhada do material selecionado na fase anterior, extraindo-se os conteúdos relevantes. Por fim, a quarta etapa envolveu a compilação dos conteúdos e dados extraídos, bem como, a escrita do artigo. Para Miguel (2007), pesquisas bibliográficas permitem identificar, conhecer e acompanhar o desenvolvimento de determinado campo de conhecimento, levantando perspectivas e sugestões para futuros trabalhos. Além da pesquisa bibliográfica no artigo foi realizada também revisão de literatura para contextualizar a temática em estudo.

\section{RESULTADOS E DISCUSSÃO}

A Tabela 1 apresenta a matriz SWOT com o resumo dos principais impactos positivos e também das limitações do PBC.

\subsection{Impactos na Educação}

Na área da educação a ação do PBC buscou fortalecer a educação infantil visando a quebra do ciclo reprodutivo da extrema pobreza e a redução das desigualdades sociais. Para isso, houve aumento da quantidade de vagas para as crianças de 0 a 48 meses nas creches públicas ou conveniadas com o poder público, especialmente para a inclusão das crianças de famílias beneficiárias do PBF (COSTA; MAFRA; BACHTOLD, 2014). Essa ação foi de extrema necessidade, pois as estatísticas educacionais da época de lançamento do PBC demonstram que ainda existiam barreiras para que a população de maior vulnerabilidade social tivesse acesso às creches para suas crianças. Para Costa, Mafra e Bachtold (2014) embora o Plano Nacional de Educação (PNE) do período de 2001 a 2010 tenha possibilitado aumento da cobertura de creches para a população de 0 a 3 anos, somente $12 \%$ dos filhos da população mais pobre conseguiam vagas na educação infantil para suas crianças de até 3 anos em 2012, porcentagem bastante pequena quando comparada aos $41,4 \%$ dos filhos dos mais ricos que tinham acesso à creche no mesmo ano. Esses dados demonstram a necessidade urgente de ações que mitiguem essa situação.

Logo após os primeiros anos de implantação do PBC é possível verificar os seus efeitos positivos na educação infantil. Esses efeitos são resultantes do repasse financeiro realizado pelo governo federal aos municípios. Entre os anos de 2012 e 2014 foram transferidos cerca de 1,48 bilhão de reais, estimulando as prefeituras a ampliar o atendimento às crianças mais pobres e melhorando a qualidade da educação de maneira geral (COSTA; MAFRA; BACHTOLD, 2014). Analisando o censo escolar de 2013 elaborado pelo Instituto Nacional de Estudos e Pesquisas Educacionais Anísio Teixeira (INEP) é possível observar um significativo aumento das matrículas de creches e pré-escolas, sendo esse resultado um reflexo positivo do reconhecimento da importância da educação infantil para o desenvolvimento futuro da criança e também dos esforços públicos para a ampliação da sua oferta. Ainda conforme o documento, no ano de 2013 foram contabilizadas mais de 7,5 milhões de matrículas na educação infantil, onde aproximadamente $36 \%$ dessas matrículas foram realizadas em creches, principalmente naquelas administradas pelos governos municipais. Também em 2013, 14\% das crianças das famílias mais pobres passaram a ter acesso a creche, no ano seguinte (2014) esse valor sobe para $19,6 \%$ o que representa um crescimento inicial acelerado e significativo, conforme Costa, Mafra e Bachtold (2014).

Nos anos posteriores à implantação do PBC as ma- 


\begin{tabular}{|c|c|}
\hline Pontos Fortes & Oportunidades \\
\hline Fortalecimento da educação infantil & Redução das desigualdades sociais \\
\hline Aumento na quantidade de vagas nas creches & Melhor acesso a creche para as crianças \\
\hline Qualificação do profissional de educação infantil & Melhora do desenvolvimento cognitivo \\
\hline Ampliação da suplementação mineral & Docentes mais capacitados \\
\hline Ampliação do Programa Saúde na Escola & Redução da anemia \\
\hline Distribuição gratuita de medicamentos para asma & Redução da internação por asma \\
\hline Benefício de superação da extrema pobreza & Redução do índice de pobreza \\
\hline $\begin{array}{l}\text { Pontos Fracos } \\
\end{array}$ & Ameaças \\
\hline $\begin{array}{l}\text { Baixa adesão da zona rural } \\
\mathrm{Na} \text { renda, os efeitos estão associados ao PBF }\end{array}$ & Interrupção e/ou redução do repasse financeiro \\
\hline
\end{tabular}

trículas em creches mantiveram-se em alta, sendo isso um reflexo positivo das ações do programa, uma vez que esse aumento ocorreu principalmente nas creches públicas. A Figura 1 apresenta a evolução no número de matrículas nas creches no período de 2015 a 2019. No censo escolar de 2019 verifica-se que houve um crescimento de $12,6 \%$ no número de matrículas da educação infantil no período de 2015 a 2019, atingindo aproximadamente 9 milhões de matrículas no último ano, sendo esse crescimento decorrente principalmente do aumento das matrículas nas creches que no período avaliado foi superior às matrículas nas pré-escolas. Ainda de acordo com o censo de 2019, a maior parte das matrículas nas creches ocorreram nas instituições municipais, podendo-se então inferir que as crianças matriculadas nesses estabelecimentos se beneficiam direta ou indiretamente do PBC. Quanto à localização, do total de matrículas em creches observado em 2019, apenas $6,7 \%$ ocorreram em instituições localizadas nas zonas rurais, demonstrando que ainda há dificuldade de acesso às creches nessas regiões e, por isso, as ações do $\mathrm{PBC}$ na educação devem ser continuadas.

Figura 1: Evolução das matrículas nas creches de 2015 a 2019.

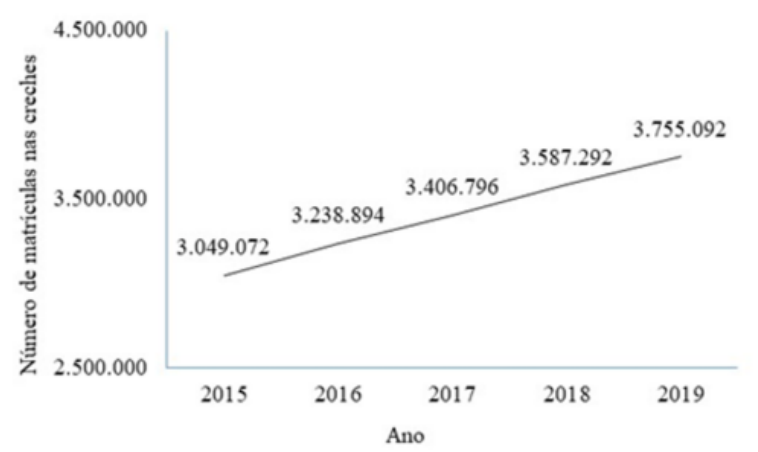

Fonte: INEP (2019).
As creches desempenham funções de grande importância para o desenvolvimento infantil. Camilli et al. (2010) verificaram que os programas pré-escolares impactam positivamente a vida das crianças, destacando os benefícios relacionados às habilidades sociais das crianças e ao seu progresso escolar. Para Burger (2010) a creche colabora com o desenvolvimento cognitivo das crianças na primeira infância por meio da promoção de experiências sociais e cognitivas complementares ao ambiente familiar. Estudos têm demonstrado que os benefícios das creches são ainda mais expressivos na população de baixa renda e escolaridade (GEOFFROY et al. 2007; HUNGERFORD; COX, 2006). Ainda nesse sentido, Burger (2010) observou em seu estudo que a educação infantil pode oferecer às crianças provenientes de contextos de baixa renda e baixa escolaridade um começo mais favorável na escola.

Além do aumento das vagas nas creches, o PBC, na educação, possibilitou também o desenvolvimento de ações complementares tais como a qualificação do profissional de educação infantil e a aquisição dos insumos necessários para o cuidado infantil de forma integral. No âmbito da educação infantil, a formação inicial e continuada dos docentes e dos profissionais que exercem atividades de suporte é de extrema necessidade. Conforme Carvalho et al. (2015) na educação infantil é imprescindível a formação apropriada do professor e demais profissionais, não sendo permitido que essa modalidade de ensino seja praticada por pessoas sem preparação. Sendo assim, ações que fomentem a qualificação para o público atuante no ensino infantil acarretam em benefícios em todo o processo de educação infantil. Desde 2015, observa-se um crescimento gradual no percentual de docentes com nível superior completo atuando na educação infantil, de 63,1\%, em 2015, para 76,3\%, em 2019 (BRASIL, 2019), esse resultado pode ser compreendido como um efeito benéfico do PBC nesse setor. As ações complementares realizadas pelo 
PBC colaboram para a manutenção do padrão de qualidade dos serviços oferecidos pelas creches.

\subsection{Impactos na Saúde}

Situações não exitosas vivenciadas ao longo da infância podem refletir na saúde, bem-estar físico e mental do indivíduo na idade adulta. Para Silva (2012), por ser uma fase dinâmica, a infância necessita de atenção e cuidado nos aspectos de saúde. Sendo assim, quando se almeja o crescimento e desenvolvimento saudável das crianças é indispensável que haja atenção e planejamento dos serviços de saúde, políticas públicas de saúde e legislação, voltada a esse público (TOMASI et al. 2011).

Tendo em vista a importância e necessidade do fortalecimento dos aspectos de saúde na erradicação e quebra do ciclo reprodutivo da extrema pobreza, o PBC direcionou ações de saúde ao público infantil visando garantir o crescimento saudável das crianças beneficiadas e assim corroborar com a melhoria da qualidade de vida das famílias assistidas. Na saúde infantil o PBC buscou agir diretamente no controle e erradicação dos principais problemas que afetam por longas datas o desenvolvimento saudável das crianças. Nesse sentido, o Ministério da Saúde, por meio do PBC, ampliou os programas de suplementação mineral passando a distribuir mais doses de vitamina A e sulfato ferroso fazendo com que um maior número de municípios de todas as regiões brasileiras fossem abrangidos o que, consequentemente, elevou a quantidade de crianças atendidas. O PBC possibilitou também a ampliação do Programa Saúde na Escola (PSE) que passou a incluir em suas ações as creches e pré-escolas. Além disso, passou a distribuir gratuitamente medicamentos para o controle da asma infantil, tendo essas ações impactado positivamente na saúde dos beneficiados.

Na suplementação mineral o PBC teve efeitos positivos, uma vez que vitamina $\mathrm{A}$ e ferro são elementos indispensáveis ao metabolismo humano e as crianças precisam deles em quantidades suficientes para alcançarem um bom desenvolvimento. Em estudo publicado em 2010, Vieira e Ferreira (2010) verificaram que a anemia, resultante da carência de ferro, representava um grave problema de saúde pública no Brasil, afetando milhões de crianças, principalmente aquelas de maior vulnerabilidade socioeconômica, ocasionando sérios danos ao seu crescimento, desenvolvimento e saúde geral. Para os autores, os gestores de políticas públicas deveriam reconhecer o controle dessa situação como uma questão de alta prioridade dos municípios, dos estados e do país. A deficiência de ferro e consequente anemia era um problema persistente em crianças em idade pré-escolar conforme observa-se em Monteiro et al. (2002). De acordo com o estudo da Pesquisa Nacional de Demografia e Saúde da Criança e da Mulher - PNDS (BRASIL, 2009) a prevalência de anemia em crianças no Brasil era de $20,9 \%$, com maior frequência nas classes sociais menos favorecidas, sendo a região Nordeste a mais acometida.

Semelhante a deficiência de ferro, Milagres, Nunes e Pinheiro-Sant'Ana (2007) observaram que a carência de vitamina A também configurava-se como um problema de saúde pública nacional acometendo principalmente o público infantil da região Nordeste. O baixo teor de vitamina A em crianças é preocupante, pois, segundo Diniz (2001) a deficiência dessa vitamina aumenta a morbidade e mortalidade das crianças. No Brasil, a prevalência da deficiência de vitamina A era de $17,4 \%$ acometendo principalmente crianças de 6 a 59 meses de idade das regiões Nordeste e Sudeste (BRASIL, 2009). A carência de vitamina A em crianças torna as mesmas mais vulneráveis a sofrer infecções mais agudas destacando-se a diarreia e as infecções respiratórias (BRASIL, 2007). Além disso, segundo a SOCIEDADE BRASILEIRA DE PEDIATRIA (2007) a deficiência de vitamina A afeta o metabolismo do ferro podendo acarretar na carência desse outro elemento a nível celular, por isso, a suplementação de vitamina A deve ser associada à suplementação de ferro.

O ferro e a vitamina A são micronutrientes que podem ser encontrados em alimentos de origem animal e vegetal. Para Jaime, Amaral e Júnior (2014) práticas alimentares inadequadas na primeira infância, consequentes da falta de acesso a alimentos em quantidade e qualidade suficientes, estão relacionadas a carências específicas desses micronutrientes. A redução da desnutrição a nível nacional não resultou na erradicação das deficiências de micronutrientes, em particular a anemia e a deficiência de vitamina A, que possivelmente estão mais associadas à qualidade da alimentação do que à quantidade de alimentos ingeridos (BRASIL, 2009). Dessa forma, os hábitos alimentares e o baixo nível socioeconômico são variáveis que estão associadas aos baixos teores de nutrientes essenciais nas crianças e o PBC auxiliou na mitigação dessa situação possibilitando um crescimento e desenvolvimento infantil mais saudável.

De acordo com Jaime, Amaral e Júnior (2014) no período compreendido entre 2012 e $2014,9,1$ milhões de crianças na faixa etária de 6 a 59 meses receberam suplementação com megadoses de vitamina A. Na suplementação de ferro, além da ampliação de sua abrangência, o PBC proporcionou também mudanças positivas na dosagem e periodicidade de aplicação. A 
dose e o período recomendados passaram de $25 \mathrm{mg}$ de ferro elementar semanalmente para $1 \mathrm{mg} / \mathrm{kg}$ diariamente, conforme Calheiros (2017). Ainda segundo a autora, a faixa etária infantil alvo da ação passou de 6 a 18 meses para 6 a 24 meses após atualização e ampliação do programa.

De acordo com o Ministério da Saúde (2020), nos últimos 13 anos, o Brasil reduziu expressivamente os casos de anemia e carência de vitamina A em crianças de até 5 anos de idade. Conforme a pesquisa os casos de anemia reduziram pela metade, recuando de $20,9 \%$ em 2006 para 10\% em 2019, já na carência de vitamina A, a redução foi ainda maior apresentando um percentual de 65,5\% menor em 2019 quando comparado ao ano de 2006. Entre os fatores responsáveis por esse resultado positivo, destacam-se as ações implementadas pelo PBC.

Ainda no contexto da suplementação mineral em crianças, o PBC possibilitou o desenvolvimento da ação NutriSUS que, segundo Costa, Mafra e Bachtold (2014), consiste na distribuição de sachês nutricionais para as creches e pré-escolas que fazem parte do Programa Saúde na Escola (PSE) e que optaram pela ação. Essa estratégia tem como objetivo a prevenção e o controle das deficiências de vitaminas e minerais na infância para que assim seja possível a potencialização do pleno desenvolvimento infantil (BRASIL, 2015). Nas creches e pré-escolas, os sachês de complementação nutricional devem ser adicionados a uma refeição diária das crianças, por um período de dois meses por ano. Vale ressaltar que o PSE só foi expandido para creches e pré-escolas após a implementação do PBC, a partir dessa expansão diversos municípios passaram a aderir ao PSE incluindo suas creches e pré-escolas. De acordo com Jaime, Amaral e Júnior (2014) após a expansão proporcionada pelo $\mathrm{PBC}$, mais de 1 milhão de educandos de creches e mais de 2 milhões de educandos de pré-escolas foram abrangidos pelo PSE, podendo-se então inferir que essa ação do PBC tem contribuído com a melhoria dos aspectos nutricionais e de saúde para milhões de crianças brasileiras.

Além das ações de suplementação mineral, a distribuição gratuita de medicamentos para o controle da asma em crianças também foi uma ação do PBC na área da saúde que se destacou positivamente. Conforme Martire (2012), a asma é uma doença inflamatória crônica, caracterizada por distúrbio ventilatório obstrutivo de graus variados, sendo uma das principais causas de visita às salas de emergência pediátrica nas unidades hospitalares. Essa inflamação representa a segunda maior causa de internação e óbito de crianças (COSTA; MAFRA; BACHTOLD, 2014), com alta prevalência e impactos em crianças e adolescentes brasileiros (SOLé et al. 2014). Após a implementação do PBC, os medicamentos para o tratamento adequado de crianças asmáticas passaram a ser disponibilizados sem custo nas farmácias pertencentes ao programa Aqui Tem Farmácia Popular (JAIME; AMARAL; JúNIOR, 2014). Por se tratar de uma inflamação crônica de alta prevalência no público infantil, o acesso gratuito à medicação recomendada corrobora positivamente para o controle da enfermidade reduzindo assim o risco de morte.

\subsection{Impactos na Renda}

Nos aspectos da renda familiar a literatura pouco aborda os efeitos exclusivos do PBC uma vez que o mesmo está associado ao PBF, fato que dificulta a sua análise isoladamente. O PBC modificou a lógica de cálculo de benefícios do PBF e essa modificação possibilitou a criação do benefício de superação da extrema pobreza (COSTA; MAFRA; BACHTOLD, 2014). Por meio desse novo benefício passou a ser repassada uma ajuda financeira adicional ao PBF para aquelas famílias com crianças em idade escolar que ainda não tinham superado a situação de extrema pobreza, sendo o valor repassado variável de acordo com a intensidade da pobreza de cada família. Para Campello e Mello (2014) a criação do benefício de superação da extrema pobreza foi motivada pela premissa de que as crianças não saem da pobreza sem que toda sua família também supere essa condição. Ainda de acordo com os autores, era necessário garantir que as famílias alcançassem um patamar mínimo de renda que permitisse que outras políticas também fossem acessadas pelos seus membros.

Conforme Costa, Mafra e Bachtold (2014), no início de 2012, ano de implantação do PBC, o valor adicional para superação da extrema pobreza passou a ser transferido inicialmente para as famílias inscritas no PBF com crianças na faixa etária de até 6 anos de idade. Os resultados foram tão promissores que, segundo os autores, no final de 2012 a ação foi estendida para as famílias com crianças de até 15 anos. Silva, Bruno e Silva (2020) analisando a pobreza multidimensional no Brasil no período de 2004 a 2015, observaram que houve redução no índice de pobreza ao longo dos anos. Em termos absolutos, os autores verificaram que 12 milhões de pessoas superaram a linha da extrema pobreza no período avaliado. Souza et al. (2019) observaram que, no ano de 2017, 3,4 milhões de pessoas deixaram de viver em pobreza extrema e 3,2 milhões foram elevadas acima da linha de pobreza, associando esse fato aos efeitos benéficos do PBF e de seus benefícios adicionais tais como o PBC.

Nesse sentido, pode-se inferir que as políticas de 
IMPACTOS DO PROGRAMA BRASIL CARINHOSO NA EDUCAÇÃO, SAÚDE E RENDA

transferência de renda tais como o PBF e PBC corroboraram para a superação da situação de extrema pobreza em muitas famílias brasileiras, sendo esse um impacto bastante positivo. No âmbito da educação infantil, Morais et al. (2015) afirmam que os programas de transferência de renda, como o PBF e PBC são importantes, pois contribuem com o desenvolvimento integral de crianças economicamente desfavorecidas no Brasil. As ações do PBC na renda das famílias devem ser continuadas principalmente no período pós-pandêmico evitando assim que um número exagerado de famílias voltem a viver economicamente abaixo das linhas de pobreza e extrema pobreza.

\section{CONSIDERAÇÕES FINAIS}

Baseado no exposto, considera-se que o PBC é uma das principais ações do BSM no combate à perpetuação da pobreza e extrema pobreza e tem corroborado para a melhoria das condições básicas de vida da população abrangida. Suas ações têm possibilitado avanços nos aspectos de educação, saúde e renda relacionados a educação infantil, impactando positivamente a vida das crianças de baixa renda, bem como, de suas famílias. Nesse sentido, o programa foi e tem sido essencial para o fortalecimento e manutenção da qualidade da educação infantil, sendo imprescindível a sua manutenção para que mais famílias e suas crianças possam ser abrangidas, principalmente nas regiões mais carentes e mais afastadas dos grandes centros urbanos.

\section{REFERÊNCIAS}

BACHTOLD, I. V. Quando o estado encontra suas margens: considerações etnográficas sobre um mutirão da estratégia de busca ativa no estado do pará. Horizontes Antropológicos, SciELO Brasil, v. 22, n. 46, p. 273-301, 2016.

BRASIL. Cadernos de atenção básica: Carências de micronutrientes. Brasília: Ministério da Saúde, 2007. Disponível em: <http://bvsms.saude.gov.br/bvs/publicacoes/cadernos_ atencao_basica_carencias_micronutrientes.pdf> Acesso em: 20 mar. 2021.

. Pesquisa Nacional de Demografia e Saúde da Criança e da Mulher. Brasília: Ministério da Saúde, 2009. Disponível em: <http://bvsms.saude.gov.br/bvs/pnds/img/relatorio_ final_PNDS2006_04julho2008.pdf>. Acesso em: 20 mar. 2021.

Lei $\mathrm{n}^{\circ}$ 12.722, de 3 de outubro de 2012. Altera as Leis $\mathrm{n}^{\circ} \mathrm{s} \mathbf{1 0 . 8 3 6}$, de 9 de janeiro de 2004,
12.462, de 4 de agosto de 2011, e 11.977, de 7 de julho de 2009; dispõe sobre o apoio financeiro da União aos Municípios e ao Distrito Federal para ampliação da oferta da educação infantil; e dá outras providências. Brasília: Diário Oficial [da] República Federativa do Brasil, 2012.

Censo da Educação Básica 2013: Resumo

Técnico. Brasília: Instituto Nacional de Estudos e Pesquisas Educacionais Anísio Teixeira (Inep), 2014. 39 p. Disponível em: <https://www.gov.br/inep/pt-br/ areas-de-atuacao/pesquisas-estatisticas-e-indicadores/ censo-escolar/resultados $>$. Acesso em: 18 mar. 2021.

Resolução $\mathbf{n}^{\circ}$ 1, de 28 de novembro de 2014. Define as despesas permitidas com recursos repassados aos municípios e ao Distrito Federal a título de apoio financeiro suplementar à manutenção e ao desenvolvimento da educação infantil, para o atendimento em creches de crianças de 0 (zero) a 48 (quarenta e oito) meses, e dá outras providências. Brasília: SEB/MEC, 2014. Disponível em: <http://www.fnde.gov.br/index.php/programas/ brasil-carinhoso/sobre-o-plano-ou-programa/ legislacao> Acesso em: 09 mar. 2021.

NutriSUS: caderno de orientações: estratégia de fortificação da alimentação infantil com micronutrientes (vitaminas e minerais) em pó. Brasília: Ministério da Saúde, 2015. 23 p. Disponível em: <http://189.28.128.100/dab/docs/portaldab/ publicacoes/caderno_orientacoes_nutrisus.pdf $>$. Acesso em: 21 mar. 2021.

Brasil Carinhoso. Brasília: Fundo

Nacional de Desenvolvimento da Educação, 2017. Disponível em: <https://www.fnde.gov.br/programas/ brasil-carinhoso > Acesso em: 08 mar. 2021.

Brasil reduz anemia e carência de vitamina A em crianças de até cinco anos. Brasília: Ministério da Saúde, 2020. Disponível em: <https://www.gov.br/saude/pt-br/assuntos/noticias/ brasil-reduz-anemia-e-carencia-de-vitamina-a-eml -criancas-de-ate-cinco-anos> Acesso em: 20 mar. 2021.

Censo da Educação Básica 2019: Resumo Técnico. Brasília: Instituto Nacional de Estudos e Pesquisas Educacionais Anísio Teixeira (Inep), 2020. 94 p. Disponível em: <https://www.gov.br/inep/pt-br/ areas-de-atuacao/pesquisas-estatisticas-e-indicadores/ censo-escolar/resultados $>$. Acesso em: 18 mar. 2021. 
IMPACTOS DO PROGRAMA BRASIL CARINHOSO NA EDUCAÇÃO, SAÚDE E RENDA

BURGER, K. How does early childhood care and education affect cognitive development? an international review of the effects of early interventions for children from different social backgrounds. Early childhood research quarterly, Elsevier, v. 25, n. 2, p. 140-165, 2010.

CALHEIROS, M. S. C. Efetividade do programa nacional de suplementação de ferro: estudo de base populacional com lactentes de 6 a 18 meses do estado de Alagoas. 71 p. Dissertação (Mestrado em Nutrição) - Universidade Federal de Alagoas, Maceió, 2017.

CAMILLI, G.; VARGAS, S.; RYAN, S.; BARNETT, W. S. Meta-analysis of the effects of early education interventions on cognitive and social development. Teachers college record, v. 112, n. 3, p. 579-620, 2010.

CAMPELLO, T.; MELLO, J. O processo de formulação e os desafios do plano brasil sem miséria: por um país rico e com oportunidades para todos. In: T, C.; T, F.; V, C. P. (Ed.). O Brasil sem miséria. Brasília: Ministério do Desenvolvimento Social, 2014. p. 33-66.

CARVALHO, D. M.; ARAúJO, S. C. F.; PINHEIRO, F. V. R.; DIAS, L. S. Educação infantil: desafios e perspectivas. in: Congresso nacional de educação. In: Anais. 12. ed. Curitiba: PUCPR, 2015. p. 14255-14269.

CÓRDOVA, I. d.; ALVES, I. G. Programas de transferência condicionada de renda na ótica dos direitos sociais: uma opção para o combate das vulnerabilidades brasileiras. Polis. Revista Latinoamericana, Centro de Investigación Sociedad y Politicas Públicas (CISPO), v. 1, n. 53, p. 111-126, 2019.

COSTA, P. V.; FALCãO, T. Coordenação intersetorial das ações do plano brasil sem miséria. In:

CAMPELlO, T.; FALCãO, T.; COSTA, P. V. (Ed.). O Brasil sem miséria. Brasília: MDS, 2014.

COSTA, P. V.; MAFRA, R. d.; BACHTOLD, I. V. O eixo de acesso a serviços e a ação brasil carinhoso no plano brasil sem miséria. In: CAMPELLO, T.; FALCãO, T.; COSTA, P. V. (Ed.). O Brasil sem miséria. Brasília: MDS, 2014.

COSTA, P. V.; MüLLER, L. H.; CARDOSO, M. M.; SOUSA, M.; LIMA, L. M. A. A estratégia de inclusão produtiva urbana no plano brasil sem miséria. In: CAMPELlO, T.; FALCãO, T.; COSTA, P. V. (Ed.). O Brasil sem miséria. Brasília: MDS, 2014.
DINIZ, A. d. S. Combate à deficiência de vitamina a: linhas de açäo e perspectivas. Revista Brasileira de Saúde Materno Infantil, v. 1, n. 1, p. 31-6, 2001.

DINIZ, S. Critérios de justiça e programas de renda mínina. Revista Katálysis, SciELO Brasil, v. 10, n. 1, p. 105-114, 2007.

FERNANDES, J. H. P. Acesso à educação e combate à desigualdade: o papel da educação no âmbito do plano brasil sem miséria. In: CAMPELLO, T.; FALCãO, T.; COSTA, P. V. (Ed.). O Brasil sem miséria. Brasília: MDS, 2014.

FONTANA, I. L. M.; CAMPOS, R. Brasil carinhoso: análises iniciais sobre a concepção de criança no programa. Revista Educativa-Revista de Educação, v. 19, n. 2, p. 737-755, 2017.

GEOFFROY, M.-C.; CÔTÉ, S. M.; BORGE, A. I.; LAROUCHE, F.; SÉGUIN, J. R.; RUTTER, M.

Association between nonmaternal care in the first year of life and children's receptive language skills prior to school entry: the moderating role of socioeconomic status. Journal of Child Psychology and Psychiatry, Wiley Online Library, v. 48, n. 5, p. 490-497, 2007.

HADDAD, A. E.; VEIGA, R.; GONçALVES, T. A.; PINTO, T. C. R. O brasil carinhoso e as políticas locais de desenvolvimento infantil - o caso do município de são paulo com o programa são paulo carinhosa. In:

CAMPELlO, T.; FALCãO, T.; COSTA, P. V. (Ed.). O Brasil sem miséria. Brasília: MDS, 2014.

HUNGERFORD, A.; COX, M. J. Family factors in child care research. Evaluation Review, v. 30, n. 5, p. 631-655, 2006.

JAIME, P. C.; AMARAL, M. A.; JúNIOR, H. M. M. Ações de saúde no plano brasil sem miséria: o acesso à saúde orientado pela superação de iniquidades. In:

CAMPELlO, T.; FALCãO, T.; COSTA, P. V. (Ed.). 0 Brasil sem miséria. Brasília: MDS, 2014.

LIMA, T. C. S. d.; MIOTO, R. C. T. Procedimentos metodológicos na construção do conhecimento científico: a pesquisa bibliográfica. Revista Katálysis, SciELO Brasil, v. 10, n. eps., p. 37-45, 2007.

LOPES, B.; AMARAL, J. N.; CALDAS, R. W. Políticas públicas: conceitos e práticas. 1 . ed. Belo Horizonte: Sebrae/MG, 2008.

MACEDO, T. S.; NOIA, A. C.; SANTOS C. E. R.A ND PINHEIRO, L. I. F. Políticas públicas e transferência de renda no brasil: análise das temáticas 
IMPACTOS DO PROGRAMA BRASIL CARINHOSO NA EDUCAÇÃO, SAÚDE E RENDA

tratadas nas pesquisas acadêmicas sobre o programa bolsa família. In: Semana do Economista \& Encontro de Egressos. 7. ed. Ilhéus: UESC, 2017. cap. Anais eletrônicos.

MARTIRE, T. M. Asma aguda na infância. Revista de pediatria SOPERJ, Revista de pediatria SOPERJ, v. 13, n. 2, p. 43-53, 2012.

MIGUEL, P. A. C. Estudo de caso na engenharia de produção: estruturação e recomendações para sua condução. Production, SciELO Brasil, v. 17, n. 1, p. 216-229, 2007.

MILAGRES, R. C. R. M.; NUNES, L. C.; PINHEIROSANT'ANA, H. M. A deficiência de vitamina a em crianças no brasil e no mundo. Ciência \& Saúde Coletiva, SciELO Brasil, v. 12, n. 5, p. 1253-1266, 2007.

MONTEIRO, C. A.; SZARFARC, S. C.; BRUNKEN, G. S.; GROSS, R.; CONDE, W. L. A prescrição semanal de sulfato ferroso pode ser altamente efetiva para reduzir níveis endêmicos de anemia na infância. Revista Brasileira de Epidemiologia, SciELO Brasil, v. 5, n. 1, p. 71-83, 2002.

MORAIS, R. L. d. S.; CARVALHO, A. M.; MAGAlHÃES, L. de C.; PINTO, P. A. F. Primeira infância e pobreza no brasil: uma análise integrada a partir de indicadores em saúde, educação e desenvolvimento social. Revista de politicas públicas, v. 19, n. 1, p. 303-314, 2015.

PASE, H. L.; MELO, C. C. Políticas públicas de transferência de renda na américa latina. Revista de Administração Pública, SciELO Brasil, v. 51, n. 2, p. 312-329, 2017.

PETRINI, J. C.; DIAS, M. C. Família no debate cultural e político contemporâneo. 2 . ed. São Paulo: Edições Loyola, 2013. 88 p.

\footnotetext{
SECRETARIA ESPECIAL DO DESENVOLVIMENTO SOCIAL. Brasil Sem Miséria. Brasília: SEDS, 2015. Disponível em: <http: //mds.gov.br/assuntos/brasil-sem-miseria/o-que-e> Acesso em: 16 mar. 2021.

SILVA, A. M. Competências da enfermeira para a atenção à criança na Rede Básica de Saúde. Dissertação (Mestrado em Enfermagem) Universidade Federal do Rio Grande do Sul, Porto Alegre, 2012.
}

SILVA, J. J.; BRUNO, M. A. P.; SILVA, D. B. N. Pobreza multidimensional no brasil: uma análise do período 2004-2015. Brazilian Journal of Political Economy, SciELO Brasil, v. 40, n. 1, p. 138-160, 2020 .

SOCIEDADE BRASILEIRA DE PEDIATRIA. Deficiência de vitamina A (documento científico). Rio de Janeiro: SBP, 2007.

SOLé, D.; CAMELO-NUNES, I. C.; WANDALSEN, G. F.; MALLOZI, M. C. A asma na criança e no adolescente brasileiro: contribuição do international study of asthma and allergies in childhood (isaac).

Revista Paulista de Pediatria, v. 32, n. 1, p. 114-125, 2014.

SOUZA, P. F.; OSóRIO, R. G.; PAIVA, L. H.; SOARES, S. Os efeitos do programa bolsa família sobre a pobreza e a desigualdade: um balanço dos primeiros quinze anos. 1. ed. Brasília: IPEA, 2019. $46 \mathrm{p}$.

TOMASI, E.; FACCHINI, L. A.; THUMÉ, E; PICCINI, R. X.; OSORIO, A.; SILVEIRA, D. S. d.; SIQUEIRA, F. V.; TEIXEIRA, V. A.; DILÉLIO, A. S.; MAIA, M. d. F. S. Características da utilização de serviços de atenção básica à saúde nas regiões sul e nordeste do brasil: diferenças por modelo de atenção. Ciência \& Saúde Coletiva, SciELO Brasil, v. 16, n. 11, p. 4395-4404, 2011.

TRONCO, G. B.; RAMOS, M. P. Linhas de pobreza no plano brasil sem miséria: análise crítica e proposta de alternativas para a medição da pobreza conforme metodologia de sonia rocha. Revista de Administração Pública, SciELO Brasil, v. 51, n. 2, p. 294-311, 2017.

VIEIRA, R. C. d. S.; FERREIRA, H. d. S. Prevalência de anemia em crianças brasileiras, segundo diferentes cenários epidemiológicos. Revista de Nutrição, SciELO Brasil, v. 23, n. 3, p. 433-444, 2010.

VIERA, M. M.; WHITAKER, M. C. O.; COSTA, Â. A.; RIBEIRO, J. M. A atenção da enfermagem na saúde da criança: revisão integrativa da literatura. Revista Brasileira Multidisciplinar, v. 18, n. 1, p. 97-115, 2015. 